\title{
Observation of optical vortex streets in walking second-harmonic generation
}

\author{
Gabriel Molina-Terriza, Dmitri V. Petrov, ${ }^{*}$ Jaume Recolons, and Lluis Torner \\ Laboratory of Photonics, Department of Signal Theory and Communications, Universitat Politecnica de Catalunya, \\ Gran Capitan UPC-D3, Barcelona ES 08034, Spain
}

Received October 16, 2001

\begin{abstract}
We report what is believed to be the first experimental observation of spontaneous nucleation of arrays of optical vortices in the process of second-harmonic generation in a crystal with moderate Poynting vector walk-off, pumped with focused beams containing screw phase dislocations. Our experiments were conducted in lithium triborate under conditions of small-efficiency second-harmonic generation pumped by nanosecond pulses at $1064 \mathrm{~nm}$. (C) 2002 Optical Society of America

OCIS codes: $190.4410,190.4420$.
\end{abstract}

Singular light beams that contain topological wave-front dislocations, are ubiquitous in optics. ${ }^{1}$ Screw dislocations, or vortices, are a common dislocation type. They are spiral phase ramps around a singularity where the amplitude of the wave vanishes and its phase is undefined. The order of the screw dislocation multiplied by its sign is referred to as the winding number, or topological charge, of the dislocation. Vortices appear spontaneously in several settings, including in speckle fields, in optical cavities, and in the doughnut laser modes. Otherwise, vortices can be readily generated, e.g., with phase masks, and nested in host beams. ${ }^{2,3}$ Light beams with nested vortices have widespread, important applications in fields as diverse as biosciences, laser cooling and trapping, micromechanics, and quantum information. ${ }^{4-8}$

By and large, the number and location of the vortices existing in a light beam at different observation planes, or foliations, ${ }^{9}$ are given by the corresponding Berry trajectories dictated by the evolution of the amplitude and wave front of the host beam. In this context, parametric wave mixing of multiple waves propagating in quadratic nonlinear media opens the door to a variety of new phenomena. Because of the parametric interaction, the waves exchange not only energy with each other but also nonlinear phase shifts and hence wave fronts. Therefore, new vortices and vortex trajectories are created in the new fields that are generated. Charge doubling in second-harmonic generation schemes, ${ }^{10,11}$ and sum- and difference-charge arithmetic operations in three-wave mixing processes, ${ }^{12}$ were demonstrated with moderate light intensities and wide pump beams. Spontaneous vortex-pair nucleation in seeded upconversion schemes ${ }^{13}$ and vortex excitation in parametric amplification from quantum noise ${ }^{14}$ constitute two additional examples of the phenomena that have been discovered. It was recently predicted that the combined effects of diffraction and Poynting vector walk-off in parametric wave-mixing processes introduce a new range of possible effects. In particular, spontaneous nucleation of multiple vortex twins whose subsequent explosion under appropriate conditions yields quasi-aligned patterns of single-charge vortices, or vortex streets, was predicted numerically. ${ }^{15}$ In this Letter we report what is believed to be the first experimental observation of such a phenomenon.

Our setup was designed with the following guidelines: First, we chose the material and input-light intensities to yield low depletion of the pump beam and hence weak interaction between the waves, and therefore changes in only the wave front of the double-frequency wave are expected. Second, numerical simulations suggest that, for a given nonlinear crystal length and input beam width, optimum conditions for creating vortex streets seem to occur with significant but moderate values of the Poynting vector walk-off. ${ }^{15}$ Therefore, a suitable nonlinear crystal and phase-matching geometry were employed. Third, theoretical predictions also suggest that a significant wave-vector mismatch between the fundamental and the second-harmonic waves can strongly affect the process of twin nucleation and subsequent dynamics as well. ${ }^{15}$ Thus, most of the experiments were conducted under conditions of effective phase matching for the conditions of the experiments, i.e., for the crystal orientation in which the generated second-harmonic beam was brightest. Notice that, for the focused beams that were employed, and because of the moderate existing walk-off, such an orientation is known to be slightly away from nominal phase matching.

The experiments were conducted in a 25 -mm-long lithium triborate (LBO) crystal cut for type I phase matching for second-harmonic generation at $\lambda=1064 \mathrm{~nm}$ in the $x-y$ plane. In this geometry, the fundamental wave propagates as an ordinary beam polarized along the $z$ axis of the crystal, the second-harmonic wave at $532 \mathrm{~nm}$ propagates as an extraordinary beam polarized in the $x-y$ plane, nominal phase matching occurs at $\varphi \simeq 11.6^{\circ}$, and the ordinary and extraordinary beams experience a moderate Poynting vector walk-off given by the angle $\rho=0.4^{\circ}$. To pump the crystal we used 8-ns pulses at $1064 \mathrm{~nm}$ from a $Q$-switched Nd:YAG laser operating at a $10-\mathrm{Hz}$ 
repetition rate. Screw phase dislocations were nested in the pump beam by use of high-diffraction-efficiency computer-generated off-axis holograms. Irises were used to select the diffraction order corresponding to single-charge vortices. Light was focused at the entrance face of the LBO crystal by lenses of different focal lengths so that the strength of diffraction and the Poynting vector walk-off could be varied. The LBO crystal was placed in a rotating holder that allowed us to tune the angle of incidence. A CCD camera was used to record images of the fundamental frequency and the second-harmonic light-intensity output of the crystal. We also acquired interferograms of the green beam output of the crystal with a reference signal generated by the laser to monitor the presence of optical vortices and to evaluate their topological charges. A delay line was used to optimize the temporal overlap between the generated and the reference pulses.

Figure 1 summarizes the central points of our observations. The top row shows the fundamentalfrequency beam output of the crystal. The pictures confirm that the propagation occurred under conditions of low depletion, so no significant changes in the input and output fundamental-frequency beams were observed. The second row shows the generated second-harmonic beams. The third row shows the corresponding interferograms with a tilted plane wave, where a characteristic fork indicates the position and the topological charge of the screw dislocations generated. $^{3}$ Location of some of the dislocations requires a close and accurate scan of the interferograms, and thus in the bottom row we show the locations and topological charges of the dislocations that we obtained in each case. In Fig. 1, the pictures are arranged so that the waist of the input beam decreases from left to right across the columns. In column (a) the input beam waist is $\sim 120 \mu \mathrm{m}$, so diffraction and walk-off effects are small, and thus the second-harmonic beam is generated with a double-charge vortex ${ }^{10,11}$ that subsequently splits into the two single-charge vortices that it contains because of walk-off and beam asymmetries. When the pump-beam waist is decreased [columns (b) and (c)] so that diffraction and walk-off can compete on a similar footing, multiple vortex twins with a zero net topological charge are continuously nucleated. Such twins split into the vortices with the positive and negative charges that they contain, which then move away from the birth point. Vortices with one topological charge move upward, and vortices with the opposite topological charge move downward. In column (b), which corresponds to a pump waist of approximately $70 \mu \mathrm{m}$, only one vortex twin was observed. Reducing the pump waist to $\sim 50 \mu \mathrm{m}$, as in column (c), reveals the generation of an additional vortex twin. In agreement with theoretical predictions,${ }^{15}$ further reductions of the beam waist destroy the vortex street, as shown in column (d). The reason for this observation is twofold. First, the strength of the walk-off relative to that of the diffraction decreases with the waist, and thus diffraction tends to dominate. Second, the smaller the beam waist, the faster the movement of the vortex twins toward the dark region, where the light intensity is very small; thus the presence of the vortices is correspondingly difficult to detect. Such observations confirm that there are optimum values of the crystal length and walk-off, and of the input beam width, for generating the vortex streets.

The pictures shown in Fig. 1 were acquired by placement of the CCD camera $25 \mathrm{~cm}$ behind the output face of the LBO crystal. Therefore, a question arises as to whether all the vortex twins that are observed were created inside the crystal. We thus placed the CCD camera at different locations beyond the crystal and found that some of the vortex twins were indeed generated outside the crystal. This result is consistent with operation in the low-depletion regime, where the pump and the generated second-harmonic beams do not interact further while propagating down the crystal. It is also in agreement with theoretical predictions that show that there is a minimum crystal length for generating vortex twins. All such observations confirm that the physical process behind the vortex-twin generation is the interference between the multiple second-harmonic beams, which is akin to a noncoaxial multiple combined beam, ${ }^{15}$ generated in the crystal as a result of the presence of walk-off.

We repeated the experiments for different operation conditions, always inside the regime of negligible pump depletion, and typically found results that were qualitatively analogous to those given above. However, we also observed that the particular patterns of output vortices that were obtained, including the (a)

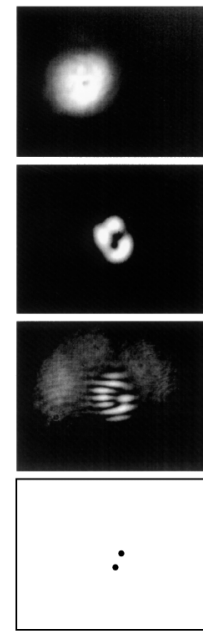

(b)

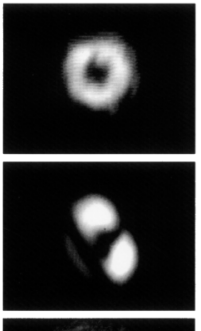

(c)

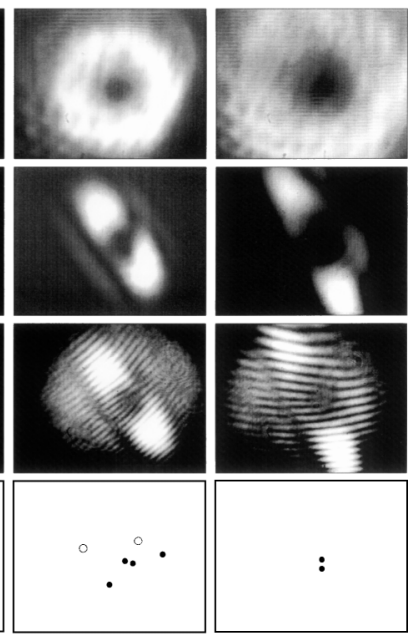

Fig. 1. Observed light distributions. Top row, fundamental-frequency pump beam after propagation inside the crystal; second row, generated secondharmonic beam; third row, interferogram of the generated second-harmonic beam with a tilted reference plane wave; bottom row, illustration of the location and charge of the optical vortices observed in the second-harmonic beam. Positive (negative) vortices, such as those in the pump beam, are shown by filled (open) circles. The pump beam was focused on the input face of the crystal with a waist of approximately (a) $120 \mu \mathrm{m}$, (b) $70 \mu \mathrm{m}$, (c) $50 \mu \mathrm{m}$, and (d) $35 \mu \mathrm{m}$. Images were taken by a CCD camera placed $25 \mathrm{~cm}$ behind the LBO crystal. The actual scale of the figures is $8 \mathrm{~mm} \times 6 \mathrm{~mm}$. The input peak power in all cases was kept at $\sim 100 \mathrm{~W}$. 
(a)

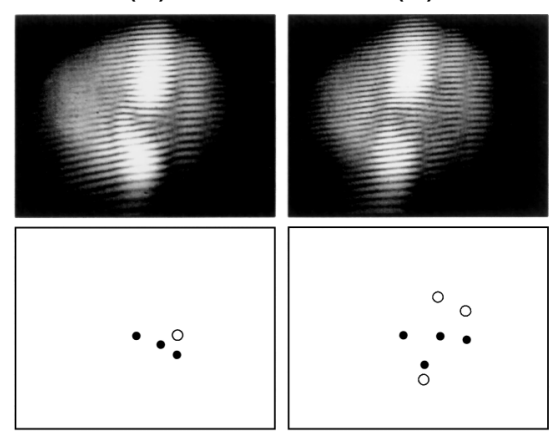

Fig. 2. (Top) interferograms of the second-harmonic beam and (bottom) locations and charges of the vortices. All features are as in Fig. 1. In (a) the waist of the input beam was located $5 \mathrm{~mm}$ in front of the LBO crystal. In (b) the crystal was rotated by a fraction of a degree relative to the orientation for highest second-harmonic-generation efficiency.

number of observable vortices, were sensitive to the details of the experimental setup. These observations are in qualitative agreement with the outcome of our comprehensive series of numerical simulations of the light evolution under the general conditions of the experiments, which showed that the pattern of vortices generated depends strongly on the exact material and input-light conditions. Such dependencies include the presence of wave-front curvatures or deviations from the ideal Gaussian shape in the pump beam. Typically illustrative examples are displayed in Fig. 2. Figure 2(a) shows the vortex pattern obtained under the same conditions as in Fig. 1(c), but now the waist of the pump beam was located $5 \mathrm{~mm}$ (which is approximately a diffraction length of the beam) outside the LBO crystal. Unlike in Fig. 1(c), nucleation of only one vortex twin was observed. Figure 2(b) shows a more extreme case. Again, this case corresponds to conditions similar to those in Fig. 1(c) but for a slightly different crystal orientation. In the case of Fig. 2(b), not only did the vortex pattern vary but also apparently at least one vortex with a positive charge moved away from the detectable region, while its sister vortex carrying the opposite topological charge remained inside the detectable region. We attribute such fast vortex drifts, which we wish to stress were also found in the numerical simulations, to the presence of ellipticities and large wave-front gradients across the pump beam. Therefore, we anticipate that the generation of clean vortex streets, closer to those predicted theoretical for ideal conditions, ${ }^{15}$ should be possible with optimized low-noise input pump signals.

We conclude by noting that vortex streets appear in a variety of natural phenomena. ${ }^{16}$ For example, in aerodynamics, periodic pressure and density fluctuations produced by the existence of Bénard-von Kármán vortex streets are responsible for the familiar whistling of thin wires in the wind or of fast-moving thin sticks in calm air. Similarly, oceanic vortex streets generated by circulating drift currents in the oceans, e.g., in the gulf stream, behind the Cozumel island off the Yucatan coast, have been recorded. Formation of such vortex streets relies on the dynamics of the viscous boundary layer at obstacles, a physical effect that is absent from our setting, in which the vortices are generated by the interference of multiple walking beams. However, our observations add to previous evidence, ${ }^{17-20}$ stressing that the analogy that exists under proper conditions between singular optics and fluid vortices is a continuously inspiring source for the discovery of new phenomena with light.

This work was supported by the Generalitat de Catalunya and by the Spanish Government under contract TIC2000-1010. We are indebted to S. Carrasco and J. P. Torres for important help in the preparation of the experiments. G. Molina-Terriza's e-mail address is molina@tsc.upc.es.

*Permanent address, Istitució Catalana de Recerca i Estudis Avançats, Pg. Lluis Companys, 2308010 Barcelona, Spain.

\section{References}

1. J. F. Nye and M. V. Berry, Proc. R. Soc. London Ser. A 336, 165 (1974).

2. V. Yu. Bazhenov, M. V. Vasnetsov, and M. S. Soskin, JETP Lett. 52, 429 (1991).

3. N. R. Heckenberg, R. McDuff, C. P. Smith, and A. G. White, Opt. Lett. 17, 221 (1992).

4. A. Ashkin, Opt. Photon. News 10(5), 41 (1999).

5. M. J. Padgett and L. Allen, Contemp. Phys. 41, 275 (2000).

6. P. Galajda and P. Ormos, Appl. Phys. Lett. 78, 249 (2001).

7. A. Mair, A. Vaziri, G. Weihs, and A. Zeilinger, Nature 412, 313 (2001).

8. G. Molina-Terriza, J. P. Torres, and L. Torner, Phys. Rev. Lett. 88, 013601 (2002).

9. I. Freund, Opt. Lett. 26, 545 (2001).

10. I. V. Basistiy, V. Y. Bazhenov, M. S. Soskin, and M. V. Vasnetsov, Opt. Commun. 103, 422 (1993).

11. J. Courtial, K. Dholakia, L. Allen, and M. J. Padgett, Phys. Rev. A 56, 4193 (1997).

12. A. Berzanskis, A. Matijosius, A. Piskarskas, V. Smilgevicius, and A. Stabinis, Opt. Commun. 140, 273 (1997).

13. D. V. Petrov and L. Torner, Phys. Rev. E 58, 7903 (1998).

14. P. D. Trapani, A. Berzanskis, S. Minardi, S. Sapone, and W. Chinaglia, Phys. Rev. Lett. 81, 5133 (1998).

15. G. Molina-Terriza, L. Torner, and D. V. Petrov, Opt. Lett. 24, 899 (1999).

16. H. J. Lugt, Vortex Flow in Nature and Technology (Krieger, Malabar, Fla., 1995).

17. M. Vaupel and C. O. Weiss, Phys. Rev. A 51, 4078 (1995).

18. J. T. Malos, R. Dykstra, M. Vaupel, and C. O. Weiss, Opt. Lett. 22, 1056 (1997).

19. D. Rozas and G. A. Swartzlander, Opt. Lett. 25, 126 (2000).

20. K. Staliunas, Phys. Rev. A 48, 1573 (1992). 